\title{
Editorial: Creating, Supporting, Sustaining and Evaluating Virtual Learning Communities
}

\author{
Xun $\mathrm{Ge}^{*}$ \\ Department of Educational Psychology \\ Jeannine Rainbolt College of Education \\ University of Oklahoma, USA \\ E-mail: xge@ou.edu
}

*Corresponding author

\begin{abstract}
This special issue is dedicated to creating, building, supporting, sustaining and evaluating virtual learning communities (VLCs) using emerging technologies. The contributors from diverse disciplines have come together to share their valuable experiences and findings through their research in the following themes: (a) instructional models, strategies, approaches for building, supporting and evaluating VLCs, (b) designing effective use of tools to promote discourse and scaffold peer interactions among members, (c) iterative processes and models of designing and evaluating VLCs; and (d) various variables concerning VLCs, such as virtual community behaviors, cultural factors, adoption patterns of tools. It is hoped that these articles will provide practical guidance and offer valuable experience to both educators and researchers who are interested in designing effective VLCs and examining various aspects of VLCs to advance our understanding of VLCs.
\end{abstract}

Keywords: Virtual Learning Communities; Web 2.0; Affordances; Participatory Culture; Peer Interactions; Peer Review; Forum Discussion; Discourse; Social Networking Tools; Collaborative Platforms; Microblogging; Strategies; Approaches; Models; Moderation; Structure; Design; Evaluation; Behaviours; Ill-Structured Problem Solving; Critical Thinking; Reflective Thinking; 21st Century Skills

Biographical notes: Dr. Xun Ge is an Associate Professor with the Program of Instructional Psychology and Technology, Department of Educational Psychology at the University of Oklahoma. She holds a Ph.D. in Instructional Systems from the Pennsylvania State University. Her research interest includes virtual learning communities, computer-supported collaborative learning, and designing learning technologies and open learning environments to support student ill-structured problem solving and self-regulated learning. Dr. Ge has published several book chapters and numerous articles in leading journals and has presented at major conferences in the field of instructional psychology and technology.

\section{Introduction}

We are living in an information-rich, digital age full of wondrous power - capabilities and possibilities afforded by communication technologies. Web 2.0 technologies have opened our eyes to a new world characterized by participatory culture of collaboration, information sharing, and knowledge generation (Bonk, 2009). Examples of Web 2.0 
include social networking sites, blogs, wikis, video sharing sites, virtual worlds, and digital object repositories. These technologies provide us with endless possibilities for creating engaging learning environments and innovative instructional experiences for learners of diverse settings, from K-12 education and higher education to corporate, government, and military training. Emerging technologies not only help us to be more productive, but also enable us to be more reflective and creative. Most importantly, technologies afford us with tools to accomplish goals that would not have been able to otherwise.

However, the potentials of the emerging Web 2.0 technologies have not necessarily been fully recognized or tapped. Just as Kushiniruk (2011) indicated, there is a lack of integration of knowledge and skills related to emerging technology in health professional education, which also speak true of other disciplines. In addition, technologies are often used to simply extend or replicate the traditional classroom teaching instead of playing a more central and transformative role in learning. This issue has become an increasing concern with many researchers and scholars involved with online learning research (Kushiniruk, 2011).

As early as in the 80s, Pea (1985) argued that technology should not be used only to amplify thinking but also to reorganize mental functioning. The features of Web 2.0 prompt us to reflect on the affordances of technologies that enhance collaboration as well as the inherent nature of participation that embraces the culture of We-All-Learn (Bonk, 2009). Web 2.0 technologies can be used to develop innovative learning environments and build virtual learning communities, aiming at motivating and engaging learners meaningfully and focusing on developing the $21^{\text {st }}$ century skills that emphasize innovation, creativity, communication, collaboration, critical thinking, decision making, and problem solving. As an effort to promote meaningful use of emerging technologies, Hwang, Chen, and Shih (2011) edited a special issue consisting of a collection of papers from Technology Enhanced Learning Conference to Asia-Pacific Conference on Technology Enhanced Learning in 2010. This special issue demonstrated a trend of moving away from the technology-enhanced learning to technology transformed learning, from focusing on factual learning to supporting students engaging in meaningful and situated learning and nurturing the development of higher-order thinking skills, including critical thinking and problem-solving.

The rising innovation and growing use of Web 2.0 technologies is a reflection of the societal needs for collaboration and community support. A learning community represents the culture of learning in which everyone is involved in a collective effort of understanding (Bielaczyc \& Collins, 1999), and a virtual community involves a combination of physical and virtual interaction, social imagination, and identity (Shumar \& Renninger, 2002). In this sense, Web 2.0 technologies provide great affordances for collaborative learning and interactions, which enable members to use their imagination for transformative learning and identity development over time.

However, using emerging technologies to build a virtual learning community (VLC) is a multifaceted innovation, and it is not without challenges. Building a VLC not only involves the use of new technologies for communication and collaboration, but also new approaches to learning and instruction and new ways of thinking about learning and instruction. For both learners and instructors it is multiple-level challenges. Subsequently, there are numerous issues to be explored, examined, investigated, and addressed; for instance, learners' perceptions, motivation and identity involved in participating a VLC, strategies and methods of designing, building, managing, supporting, and evaluating 
VLCs in developing students' critical thinking, problem solving, and creativity, and teachers' beliefs about participatory culture of a VLC.

Building on the previous two special issues of Knowledge Management \& ELearning: An International Journal - Issue 1 of Volume 3 titled "Advances in Health Education Applying E-learning, Simulations and Distance Technologies" edited by Kushiniruk (2011) and Issue 3 of Volume 3 titled "Shifting from Technology-enhanced Learning to Technology-transformed Learning - Best Papers Selected from the Conference APTEL 2010" edited by Hwang et al. (2011), this special issue is dedicated to the building of VLCs using emerging technologies. In this special issue, a VLC is set in the context of both informal educational setting, such as one that supports ongoing professional development, and formal educational setting, such as one as found in a formal class setting. In response to the call for the special issue, a good number of manuscripts were received, of which 11 were selected to be included in this special issue. This edition reflects an interesting collection of papers from authors of diverse disciplines and professions (e.g., education, engineering, and healthcare), who have come together to offer their research findings and share their best practices in using Web 2.0 to build and enhance VLCs.

\section{Preview of Papers}

As a guest editor, I am very impressed by the quality and the diversity of this edition of articles, from which four major themes emerged. Below I discuss each of the themes and the interconnections of the articles among themselves and to the themes.

The first theme is the exploration of the impact of support mechanisms and social roles of instructors and peers in structured VLCs in supporting learners' reflective thinking, critical thinking, and professional identity development. Law, Ge, and Eseryel (2011) examined the development of novice instructional designers in a reflective learning community, particularly dynamic interactions of individual and group characteristics in knowledge construction process and the influence of social interactions influencing the development of epistemic frames of instructional design. Like Law et al. (2011), Ruan and Griffith (2011) also provided a protocol to facilitate in-service teachers' in-depth discussions and reflective thinking of their professional development through effective forum discussions. Zhang and Toker (2011) investigated the dynamic impacts of instructor moderations and peer reviews, as well as the respective roles of the instructor and peers in promoting students' critical thinking over a period of semester.

The second theme focuses on collaborative tools and effective strategies for using those tools, including social networking tools (e.g., Twitter), to sustain and strengthen VLC through timely peer interactions and to encourage community members to engage in meaningful discourse to support reflective thinking, situated learning and educator professional development. Wakefield, Warren, and Alsobrook (2011) examined the design of a course that utilized the real-time information network Twitter to stimulate reflective thinking through encouraging discourse among students and time on task by following guidelines set forth in the theory of Learning and Teaching as Communicative Actions. Hsu and Ching (2011) also investigated the impact of the design of the microblogging activities by using a microblogging tool (e.g., Twitter) to allow quick peer interactions to build the momentum of social learning in the VLC. Further, Ruberg and her colleagues (2011) studied the impact of e-collaborative tools and strategies in helping educators of science, technology, engineering, and mathematics (STEM) to create a VLC 
that would enable them to discuss ways they could improve their instruction and their students' performance in STEM domains.

The third theme evaluates the design of and evaluates the evolvement VLCs over collaborative learning platforms across institutions through longitudinal studies over a period of two to six years. For instance, Avila and her colleagues (2011) examined how an evaluation plan of VLC had evolved over six years and discussed how discussion forums had provided global health practitioners with a platform to exchange evidencebased information and knowledge with colleagues working around the world. In a longitude study over a period of two years, vom Brocke (2011) reported multiple case studies on the effects of a VLC, which was designed to foster so-called "agile communication skills" through a virtual space where members from different countries are brought together to collaborate.

Finally, the fourth theme is the exploration of patterns of tool use and adoption, virtual community behaviors, social cultural factors and other factors influencing VLC tools and various factors influencing the development of VLCs. The findings serve to explain some of the VLC behaviors found in the other papers or provide implications for further design and research on virtual learning communities. Malik and his colleagues (2011) investigated the user adoption patterns of HUB platforms that were designed to create and support the VLC over a period of time. Yong, Sachau, and Lassiter (2011) developed a measurement for examining the kinds of behaviors that constitute virtual community citizenship behaviors (VCCB) and testing three factors that might influence community members' willingness to engage in VCCB. Lastly, Olaniran (2011) argues that successful learning in VLCs require cultural sensitivity; and therefore he presented a conceptual paper arguing about the method to address cultural challenges of VLCs.

\section{Implications for Practice and Research}

This edition of articles is published in response to the emerging need to address the use of Web 2.0 technologies to build, support, sustain, and evaluate virtual learning communities. The articles were contributed by a group of authors from diverse disciplines, who have shared valuable experiences and findings through their research in the following areas: (a) instructional models, strategies, approaches for building, supporting and evaluating VLCs, (b) designing effective use of tools to promote discourse and scaffold peer interactions among members, (c) iterative processes and models of designing and evaluating VLCs, and (d) various factors concerning VLCs, such as VLC behaviors, cultural factors, adoption patterns of tools. It is hoped that these articles will provide practical guidance to educators who are interested in various aspects of VLCs, in both school and non-school settings, or formal and informal learning settings.

Meanwhile, this special issue also demonstrates a variety of research methods employed to capture data for investigating VLCs that yielded interesting findings worthy for researchers to examine, including case studies, mixed methods, and longitudinal studies, just to mention a few. In addition, this edition also calls for rigorous research methodology to further investigate many VLC issues (e.g., emergent leadership, identity development, VLC behaviors) to help us understand VLC phenomena and their impact on knowledge construction and community building. 


\section{Acknowledgements}

I would like to thank the Editor-in-Chief, Dr. Maggie M. Wang, for her encouragement and continuous support of the Special Issue. My thanks also go to those anonymous reviewers who have contributed their time and effort to provide constructive feedback to the authors and ensure the quality of this edition.

\section{References}

1. Avila, M., Nallathambi, K., Richey, C., \& Mwaikambo, L. (2011). Six Years of Lessons Learned in Monitoring and Evaluating Online Discussion Forums. Knowledge Management \& E-Learning: An International Journal, 3(4), 621-643.

2. Bielaczyc, K., \& Collins, A. (1999). Learning communities in classrooms: A reconceptualization of educational practices. In C. M. Reigeluth (Ed.), Instructionaldesign theories and models: A new paradigm of instructional theory (pp.269-292). New Jersey: Lawrence Erlbaum.

3. Bonk, C.J. (2009). The world is open: How Web technology is revolutionizing education. Jossey-Base.

4. Hsu, Y.C., \& Ching, Y.H. (2011). Microblogging for Strengthening a Virtual Learning Community in an Online Course. Knowledge Management \& E-Learning: An International Journal, 3(4), 585-598.

5. Hwang, G.J., Chen, N.S., \& Shih, J.L. (2011). Editorial: Shifting from technologyenhanced learning to technology-transformed learning - Best papers selected from the conference APTEL 2010. Knowledge Management \& E-Learning: An International Journal, 3(3), 306-309.

6. Kushiniruk, A.W. (2011). Editorial: Advances in health education applying Elearning, simulations and distance technologies. Knowledge Management \& ELearning: An International Journal, 3(1), 1-4.

7. Law, V., Ge, X., \& Eseryel, D. (2011). An Investigation of the Development of a Reflective Virtual Learning Community in an Ill-Structured Domain of Instructional Design. Knowledge Management \& E-Learning: An International Journal, 3(4), 513-533.

8. Malik, Q.H., Perova, N., Hacker, T.J., Streveler, R.A., Magana, A.J., Vogt, P.L., \& Bessenbacher, A.M. (2011). Creating a Virtual Learning Community with Hub Architecture: CLEERhub as a Case Study of User Adoption. Knowledge Management \& E-Learning: An International Journal, 3(4), 665-681.

9. Olaniran, B.A. (2011). Designing Functional Virtual Learning Communities Using the Bola Ola Method. Knowledge Management \& E-Learning: An International Journal, 3(4), 697-710.

10. Pea, R.D. (1985). Beyond amplification: Using the computer to reorganize mental functioning. Educational Psychologist, 20(4), 167-182.

11. Ruan, J., \& Griffith, P.L. (2011). Supporting Teacher Reflection through Online Discussion. Knowledge Management \& E-Learning: An International Journal, 3(4), 548-562. 
12. Ruberg, L.F., Cummings, M., Piecka, D.C.B., Ruckman, C., \& Seward, R. (2011). A Logical Approach to Supporting Professional Learning Communities. Knowledge Management \& E-Learning: An International Journal, 3(4), 599-620.

13. vom Brocke, C. (2011). How to Leverage Virtual Learning Communities for Teaching Agile Communication Skills? The eGroups Case at the University of Muenster in Germany and Massey University in New Zealand. Knowledge Management \& E-Learning: An International Journal, 3(4), 644-664.

14. Wakefield, J.S., Warren, S.J., \& Alsobrook, M. (2011). Learning and Teaching as Communicative Actions: A Mixed-Methods Twitter Study. Knowledge Management \& E-Learning: An International Journal, 3(4), 563-584.

15. Yong, L., \& Sachau, D., \& Lassiter, A. (2011). Developing a Measure of Virtual Community Citizenship Behavior. Knowledge Management \& E-Learning: An International Journal, 3(4), 682-696.

16. Zhang, K., \& Toker, S. (2011). Stimulating Critical Thinking in a Virtual Learning Community with Instructor Moderations and Peer Reviews. Knowledge Management \& E-Learning: An International Journal, 3(4), 534-547. 